LA-UR-02-3696

Approved for public release; distribution is unlimited.

\section{Title: $\mid$ Ion Confinement using a Self-Biased Target in Multiple Pulse Radiography}

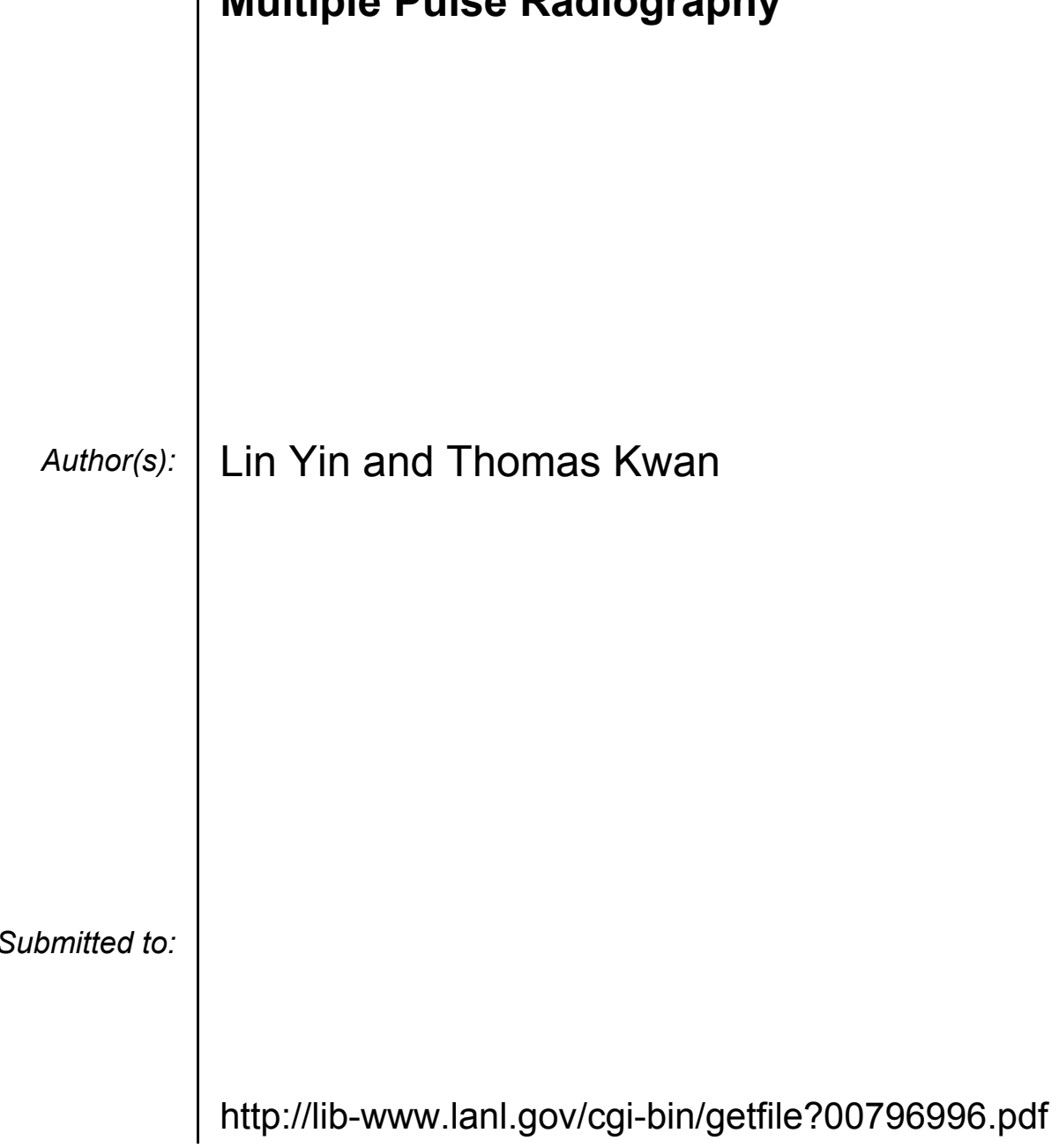




\title{
Ion Confinement using a Self-Biased Target in Multiple Pulse Radiography
}

\author{
Lin Yin and Thomas Kwan \\ Applied Physics Division, Plasma Physics Group (X-1) \\ Los Alamos National Laboratory, Los Alamos, NM 87545
}

\begin{abstract}
When a high current relativistic electron beam deposits its energy on a high-Z target, bremsstrahlung photons are produced which can be used to generate radiographs of a dynamically evolving object. This intense energy deposition can create a plasma ion plume from the converter target, however. The plasma ions cause partial charge neutralization of the electron beam and increase the beam spot size on the target, and thus affect the spatial resolution of radiographic images. Previous studies have demonstrated that a self-biased target technique can establish an electric potential between the target and the collimator that confines the plasma ion column and thereby maintains a small beam spot size [1]. In this work we use a two-dimensional particle-in-cell code to further examine the possibility of the ion confinement by the self-biased potential for DARHT-II multiple-pulse radiography. It is shown that by using the electrons deposited on the beam dump between pulses a continuous current flow can be maintained through a resistor to the ground, and the biased potential can be kept steady throughout the entire multiple pulse operation. In the simulation, the resistor is modeled by a vacuum diode. The effects of the impedance associated with the diode on the desired value of the biased potential and on its decay time are examined. In comparison to the proposed barrier foil method [2], this electromagnetic confinement avoids the possibility of ion emission from the barrier foil [3] which can cause the beam spot to grow.
\end{abstract}




\section{Introduction}

A high current relativistic electron beam with a small beam spot size on the converter target is desirable for the generation of high resolution radiographs of a dense object. However, due to the intense energy deposition by the high current electron beam, the target material can be vaporized rapidly and the resulting plasma ion column can expand upstream and partially charge neutralize the electron beam. This charge neutralization causes the electron beam to focus before reaching the target and leads to the growth of the beam spot on the target. In order to maintain the electron beam spot size, methods of ion confinement have been examined in previous studies [1] [2]. It has been demonstrated using the two-dimensional particle-in-cell code MERLIN that an electric potential between the target and the collimator can be established to confine the ion column using a self-biased target design for the single-pulse operation of the DARHT first axis [1].

The essence of the self-biased target design is illustrated in Ref. [1] (Fig. 2). As the beam electrons deposit charge on the converter target, an electron current can flow from the target to the ground through a resistor. This produces a potential drop and biases the target negatively with respect to the collimator. With an appropriately chosen resistance, the ions can be trapped effectively in the potential well and remain confined axially.

For the DARHT second axis, there are four pulses with desired lengths of 20, 20, 26, and $60 \mathrm{~ns}$. To maintain a steady, small beam spot size on the converter target for all four pulses, ions emitted from the target must be confined axially for the entire multi-pulse operation. Here, the biased potential method proposed previously for the single-pulse operation of the DARHT first axis [1] is extended for the DARHT second axis using a self-biased target and beam dump design: During the operation of each pulse, a biased potential is employed between the target and the collimator using the electron current flow from the target to the ground through a resistor. During the interval between 
successive pulses when the beam is deflected to a beam dump, one can draw an electron current from the beam dump to the ground through a resistor and set up a biased potential between the beam dump and the collimator. If the target and the beam dump are kept at the same potential, a steady biased potential can be maintained between the target and the collimator and thus the ion column can remain confined throughout the entire four-pulse operation.

In our study, the self-biased target and beam dump method is investigated with the two-dimensional particle-in-cell code MERLIN. We use an electron beam of $4 \mathrm{kA}$ instead of $2 \mathrm{kA}$ to demonstrate the effectiveness of the electromagnetic confinement in high current operation. In contrast, the barrier foil method would encounter increasing difficulties because of the ion emission when the electron beam current exceeds $2 \mathrm{kA}$. It

is shown that the method is applicable for the DARHT multiple pulse operation. The effects of the impedance associated with the vacuum diode on the value of the biased potential are examined and the effects of the impedance on the potential decay time is discussed.

\section{Ion Confinement using a Self-Biased Target and Beam Dump Design}

Two-dimensional particle-in-cell simulations are performed to examine the effectiveness of the self-biased target and beam dump method for the ion confinement. In the simulation, the beam electron energy is $20 \mathrm{MeV}$ and the beam current is $4 \mathrm{kA}$ with a rise time of 3.3 ns. The short rise time is chosen for computational efficiency. In the simulation, an electron beam is converged onto the target to produce a spot with a 2-mm diameter. The convergent angle is $6.9^{\circ}$ and the target is a $1-\mathrm{mm}$ thick tantalum foil. The beam electrons are cold and are injected from the left boundary. As the beam encounters the target, it is partially transmitted and partially reflected while a fraction of the beam electrons remain inside the target. The initial velocity vectors of the transmitted and 
reflected electrons at the target are obtained from Monte Carlo transport calculations for the $20 \mathrm{MeV}$ electron beam using the two-dimensional code CYLTRAN. According to the calculations, about $67.6 \%$ of the beam electrons are transmitted and $8.3 \%$ are reflected.

As the target is bombarded by the intense electron beam, ions begin to emit from the target surface. The electric field threshold for the ion emission from the target material is set to $100 \mathrm{kV} / \mathrm{cm}$. The space-charge-limited model is used for the ion emission and the emitted ions have no initial momentum. The ions in the simulation are assumed to be hydrogen ions (with real mass ratio $m_{i} / m_{e}=1836$ ) to shorten the computational time. The aforementioned resistor in the biased target design is modeled by a vacuum diode in the simulation. For the diode electron emission, the electric field threshold of the conducting material is chosen to be $10 \mathrm{kV} / \mathrm{cm}$, and the emission model is the same as that for the ions emitted from the target.

For the DARHT second axis, there are four pulses with lengths 20, 20, 26, and $60 \mathrm{~ns}$. During the interval between the pulses, the beam is deflected to a beam dump. In the simulation the first pulse length is set to $14.85 \mathrm{~ns}$, followed by a time interval when the target is removed. During this time the beam electrons are allowed to travel farther to the space on the right to strike the beam dump (the same material is used for the target and the dump) to mock up the deflection of the beam to the beam dump in DARHT operation.

The configuration of the simulation is shown by the top panel in Figure 1 where $z$ and $r$ are the axial and radial coordinates, and the axial and radial extent of the simulation are $32.0 \mathrm{~cm}$ and $2.5 \mathrm{~cm}$. The target and the beam dump are located at $z \simeq 8 \mathrm{~cm}$ and $z \simeq 24 \mathrm{~cm}$, respectively, and they are connected by conducting material to the diode which is located near the right boundary. The red curve on the left is the collimator. The scatter particle plot in the top panel displays the beam electrons (red), the transmitted (cyan) and reflected (magenta) electrons, the ions (green) emitted from the target, and the diode electrons (yellow) at a time $t=14.85 \mathrm{~ns}$ just before the target is removed. 
The electron current flowing through the diode gap produces a biased potential between the target and the collimator. At this time the biased potential is established and reaches a value around $340 \mathrm{kV}$. The superposition of this potential drop and the space charge potential of the particles forms a potential well for the ions, as is apparent from the beam electron energy profile along the axis shown by the second panel in Figure 1 (energy is in $\mathrm{MeV}$ ). The beam electrons lose their energy when they encounter a potential barrier and the energy difference between the beam injection boundary and the target is consistent with the value of the established biased potential at this time. As the ions are emitted from the target, they fall into the potential well and are confined axially. The ion axial momentum and energy profiles along the axis are displayed by the third and fourth panel (the momentum $p_{1}$ is normalized to $m_{i} c$, where $c$ is the speed of light, and energy is in $\mathrm{MeV}$ ). Ion trapping by the potential well is seen. Although some ions escape and travel axially upstream, the majority of the ion charge remains confined, as shown by the charge distribution profile in the bottom panel.

Between times $t=14.85 \mathrm{~ns}$ and $t=29.7 \mathrm{~ns}$ we removed the target to allow the beam electrons to travel farther to the beam dump. The configuration is shown by the top panel in Figure 2 where the scatter particle plot at $t=29.7 \mathrm{~ns}$ displays the ions emitted from the beam dump (green), as well as the transmitted (blue) and reflected (orange) electrons. Due to the electron deposition on the beam dump, electron current flows continuously through the diode gap and thus the biased potential applied at the ion column previously emitted in front of the target is well maintained. As a consequence, the charge distribution of the ions previously emitted from the target is mostly confined axially, as shown by the middle panel. Some ions appear farther upstream but the fraction of the escaped ion charge is relatively small compared to that of the confined ions. In the upstream region $(z<8 \mathrm{~cm})$, the axial electric field points in the direction of the target, as shown by the axial electric field profile along the axis at $r=0.5 \mathrm{~cm}$ in the bottom panel. As the ions emitted initially near the axis arrive in this region they move towards the target under the electric force and can be re-absorbed by the target. 
After $t=29.7 \mathrm{~ns}$ we switched off the beam dump and turned the target back on and ran the simulation until $t=46.2 \mathrm{~ns}$. The scatter particle plot is displayed by the top panel in Figure 3. The newly emitted ions from the target after $t=29.7 \mathrm{~ns}$ are shown in blue. The charge distributions of the previously emitted ions (middle panel) and the newly emitted ions (bottom panel) from the target indicate that the column length of the ions in front of the target is short and that good ion confinement is achieved by the biased target and beam dump method.

The time history of the biased potential between the target and the collimator for the entire run is displayed in the top panel in Figure 4. It is clear that, once established, the biased potential remains at a relatively steady value just below $400 \mathrm{kV}$ during the switching between the target and the beam dump. The evolution of the beam spot size, the root mean square (rms) beam radius on target, is shown in the bottom panel. The rms radius of the electron beam on the target does not exceed $0.5 \mathrm{~mm}$ during the run and it reaches a steady value below $0.4 \mathrm{~mm}$ during the second pulse, which is smaller than the rms radius of $0.8 \mathrm{~mm}$ when no ions are emitted from the target [1].

\section{Effects of the Vacuum Diode Impedance on the Biased Potential Value}

The impedance of the diode is a function of location of the diode electron emission and the gap spacing. By adjusting where the diode electron emission occurs in the simulation, one can vary the impedance associated with the vacuum diode and can select and control the biased potential between the target and the collimator. In an experiment, a resistor can be used instead to adjust the resistance for the same purpose [4]. It has been shown that to optimize the operation the beam spot size on the target can be kept to a minimum by selecting a biased potential to confine the length of the ion column [1]. In Figures 5, 6 , and 7 , the diode voltage $(\mathrm{kV})$, current $(\mathrm{kA})$, and impedance $(\Omega)$ for electron emission at $r \sim 0.21 \mathrm{~cm}, r \sim 0.61 \mathrm{~cm}$ (which is used for the simulation run presented above), 
and $r \sim 1.67 \mathrm{~cm}$ are displayed for comparison. While the diode current in all three cases reaches a value around $4 \mathrm{kA}$, which is the injected electron beam current, the diode voltage and impedance increase with decreased electron emission location $r$. Thus, the diode location can be used as a knob to adjust and select the voltage to optimize the ion confinement.

\section{Discussion and Conclusions}

We have examined the concept of a biased target and beam dump design for the confinement of the target ion plasma by the self-biased potential. Two-dimensional particlein-cell simulations have demonstrated the effectiveness of this method for the DARHT multiple pulse operation: Through the use of a potential drop produced by electron current flowing across a diode gap, a self-biased potential between the target and the collimator can be established as the beam interacts with the target and maintained when the beam is deflected to the dump. The location of the diode gap can be adjusted to select a desired value for the biased potential to achieve ion confinement.

Without the use of the beam dump to maintain the biased potential, the biased potential established during the operation of the first electron pulse gradually decays with time. The decay time depends on the impedance of the vacuum diode. Figure 8 shows how the decay time changes with the location of the diode gap. From the top to the bottom panel, the location of diode electron emission is set to around $r \sim 0.21 \mathrm{~cm}$, $r \sim 0.61 \mathrm{~cm}$, and $r \sim 1.87 \mathrm{~cm}$. The electron beam is turned off at time $t=13.2 \mathrm{~ns}$ in these runs when the biased potential is established. The decay time increases as the radial location of the diode gap decreases. The run with the smallest $r$ results in the largest diode voltage (nearly $800 \mathrm{kV}$ ) and the longest decay time (see top panel).

The ion plasma in front of the target may be confined during the interval between pulses without the use of the beam dump to maintain the biased potential if the diode voltage exceeds a threshold value. Results of a test run are discussed below in which the 
diode gap is located around $r \sim 0.21 \mathrm{~cm}$ and the injection of the electron beam stops at a time $t=13.2 \mathrm{~ns}$ as the biased potential reaches nearly $800 \mathrm{kV}$. The ions emitted from the target at time $t=13.2$ ns are confined well by the biased potential, as shown by the top panel in Figure 9. The axial momentum profile (second from top) indicates that the ions located further upstream have velocities pointing towards the target (in contrast to the results shown in Figure 1 in which the biased potential is below $400 \mathrm{kV}$ ). During the decay of the biased potential when the beam is absent, the ions left in front of the target all have velocities pointing towards the target, as seen in the third panel from the top $(t=14.0 \mathrm{~ns})$. As time goes on, these ions are re-absorbed by the target, as indicated by the bottom panel $(t=16.5 \mathrm{~ns})$. However, because of the large electric field associated with the high potential, experiments are difficult to perform under such conditions. Nevertheless, the run presents an interesting phenomenon: There appears to be a threshold value for the biased potential above which ions in front of the target can be re-absorbed by the target.

We have performed our simulations based on a $4 \mathrm{kA}$ beam current and a beam energy of $20 \mathrm{MeV}$. Previous study has shown that optimal values of the self-biased potential can be selected to minimize the beam spot size for operations with different beam currents and beam energies [1].

In our simulation study the ions are assumed to be hydrogen ions to shorten the computational time and they are singly ionized. For higher atomic number (and singly ionized) ions emitted from the target, the time it takes the ion column to axially expand into the beam and degrade the beam spot size is expected to increase [5] and the effects on the beam spot size are expected to be less significant. Thus, the self-biased potential method should still be effective in confining higher atomic number ions from the target. 


\section{Acknowledgments}

This research was performed under the auspices of the U.S. Department of Energy by the Los Alamos National Laboratory. We thank C. M. Snell for assistance in converting the encapsulated postscript files.

\section{References}

[1] T. J. T. Kwan, C. M. Snell, and P. J. Christenson, Phys. Plasmas 7, 2215 (2000).

[2] T. J. T. Kwan and C. M. Snell, Review of Scientific Instruments 71, 1363 (2000).

[3] C. Vermare et al., Los Alamos National Laboratory Report LA-UR-013220 (2001).

[4] T. J. T. Kwan et al., in Proceedings of LINAC98 Conference, Chicago, IL (NTIS, Springfield, VA, 1998), pp. 660-662.

[5] D. R. Welch and T. P. Hughes, Laser Part. Beams 16, 285 (1998). 

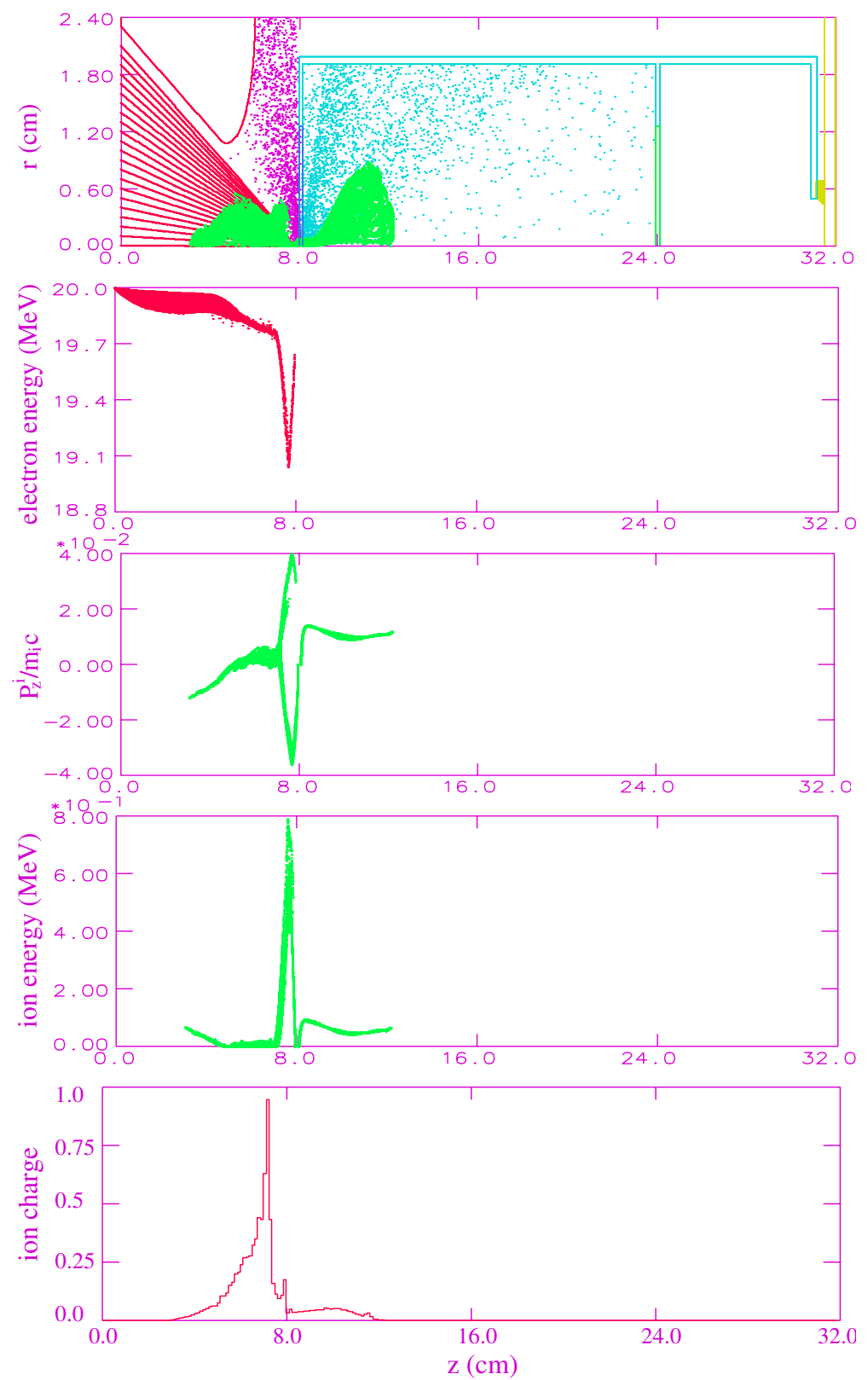

Figure 1: The configuration of the simulation and ion confinement at $t=14.85$ ns when the biased potential is established. The target and the beam dump are located at $z \simeq 8 \mathrm{~cm}$ and $z \simeq 24 \mathrm{~cm}$. From top to bottom: scatter particle plot displaying the beam electrons (red), the transmitted (cyan) and reflected (magenta) electrons, the ions (green) emitted from the target, and the diode electrons (yellow); beam electron energy profile along the axis; ion axial momentum profile showing ion trapping; ion energy profile; and ion charge distribution. 

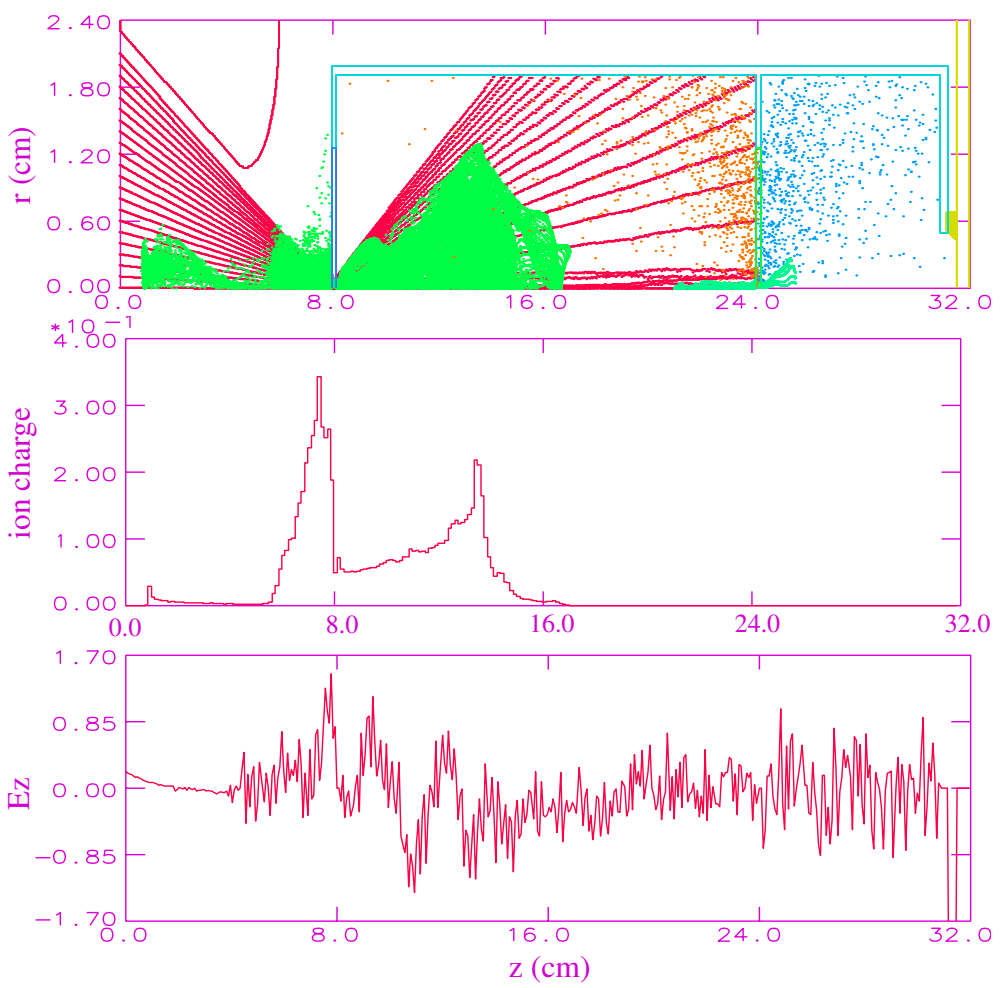

Figure 2: The simulation at $t=29.7 \mathrm{~ns}$ when beam electrons are interacting with the dump to maintain the biased potential. From top to bottom: the ions emitted from the dump (green), and the transmitted (blue) and reflected (orange) electrons; charge distribution of the ions previously emitted from the target; the axial electric field profile along the axis at $r=0.5 \mathrm{~cm}$ indicating that ions may be re-absorbed as they move towards the target under the electric force. 

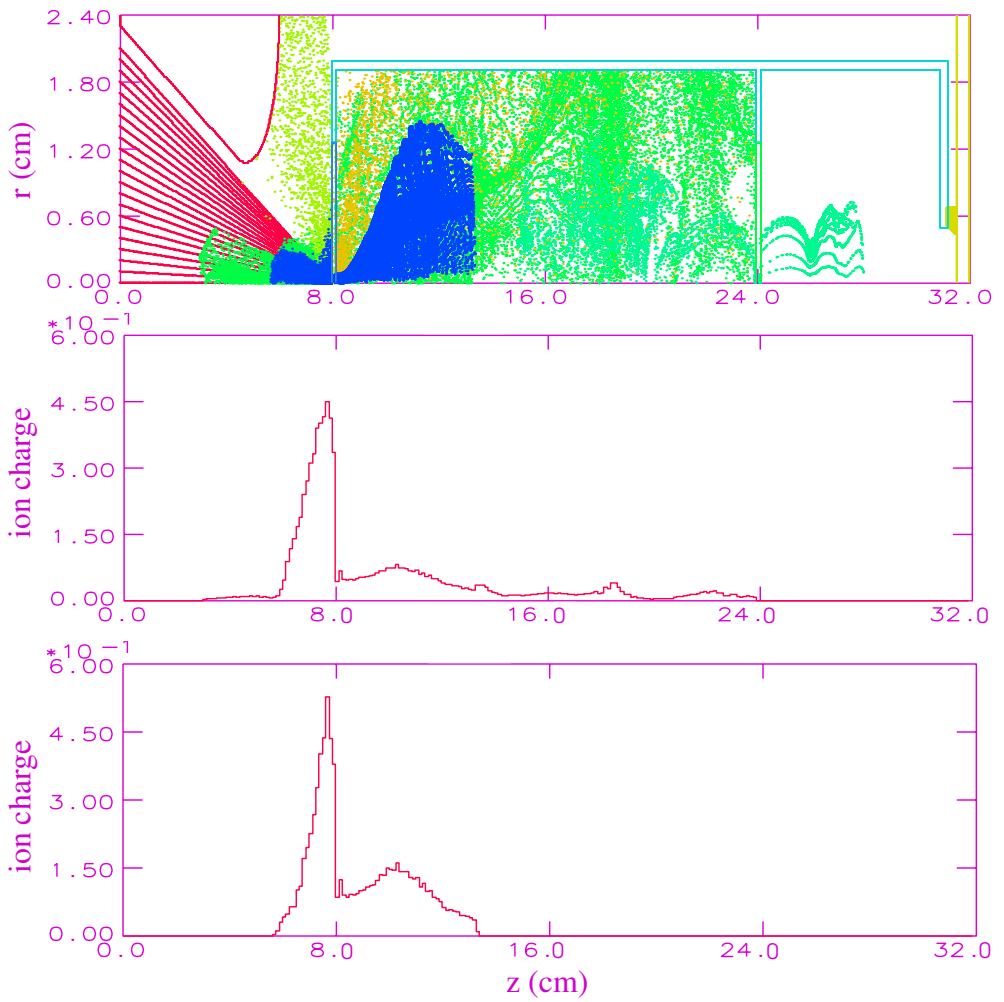

Figure 3: The simulation at $t=46.2$ ns when the second pulse is in operation. The newly emitted ions from the target are shown in blue (top). The charge distributions of the previously emitted ions (middle) and the newly emitted ions (bottom) from the target indicate that good ion confinement is achieved by the biased target and beam dump method. 

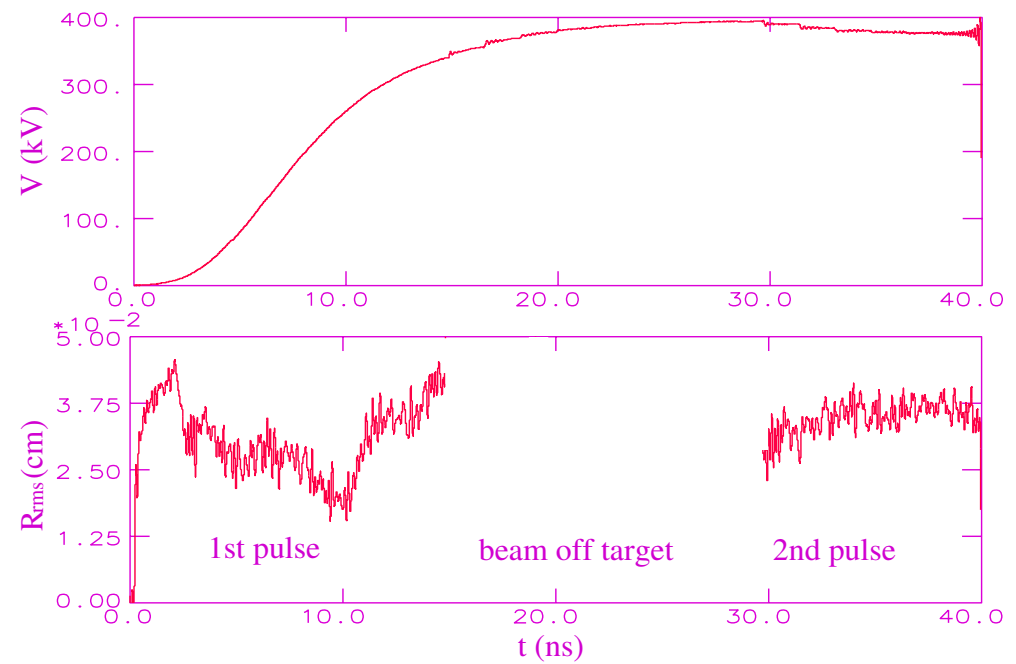

Figure 4: The time history for the entire run (top) showing that the biased potential remains at a steady value during the interval between pulses. The evolution of the beam spot size on target (bottom): the rms radius of the electron beam on the target does not exceed $0.5 \mathrm{~mm}$ during the run and it reaches a steady value below $0.4 \mathrm{~mm}$ during the second pulse. 

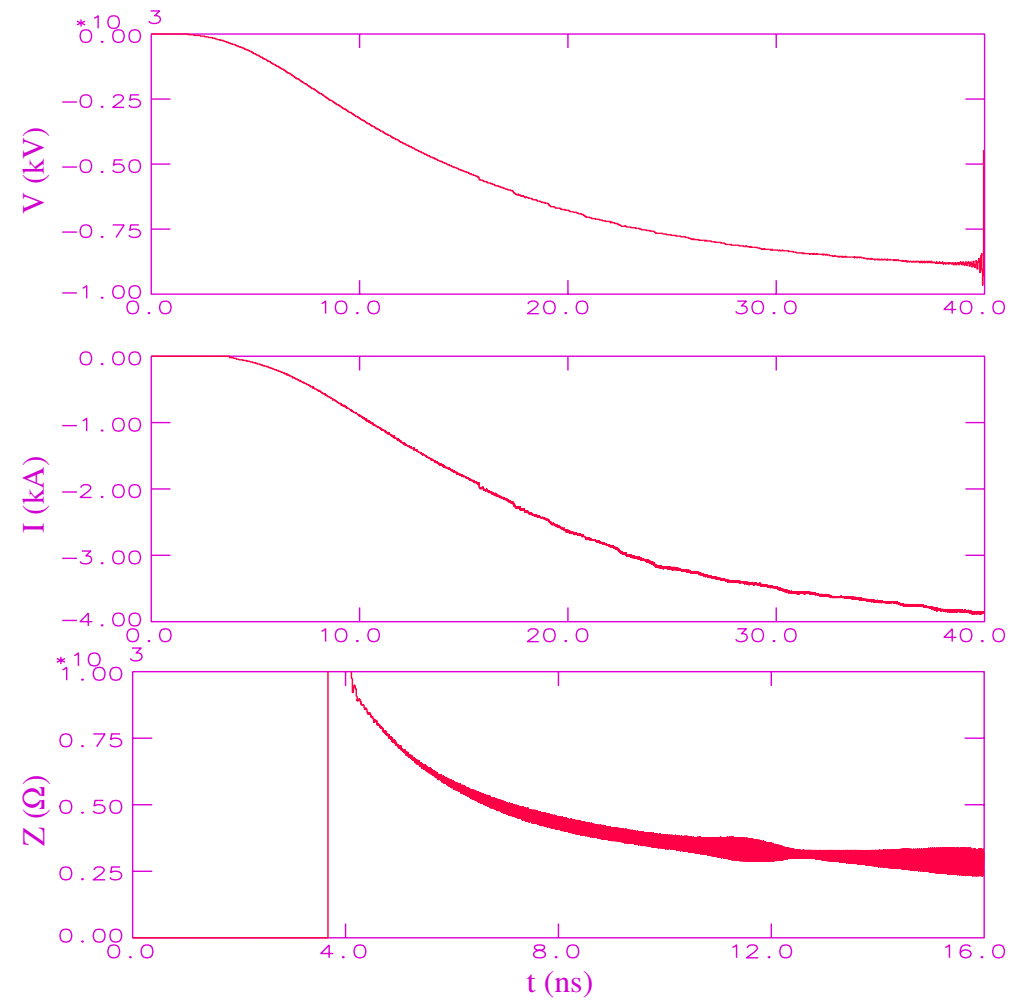

Figure 5: The diode voltage, current, and impedance for diode electron emission at $r \sim 0.21 \mathrm{~cm}$. 

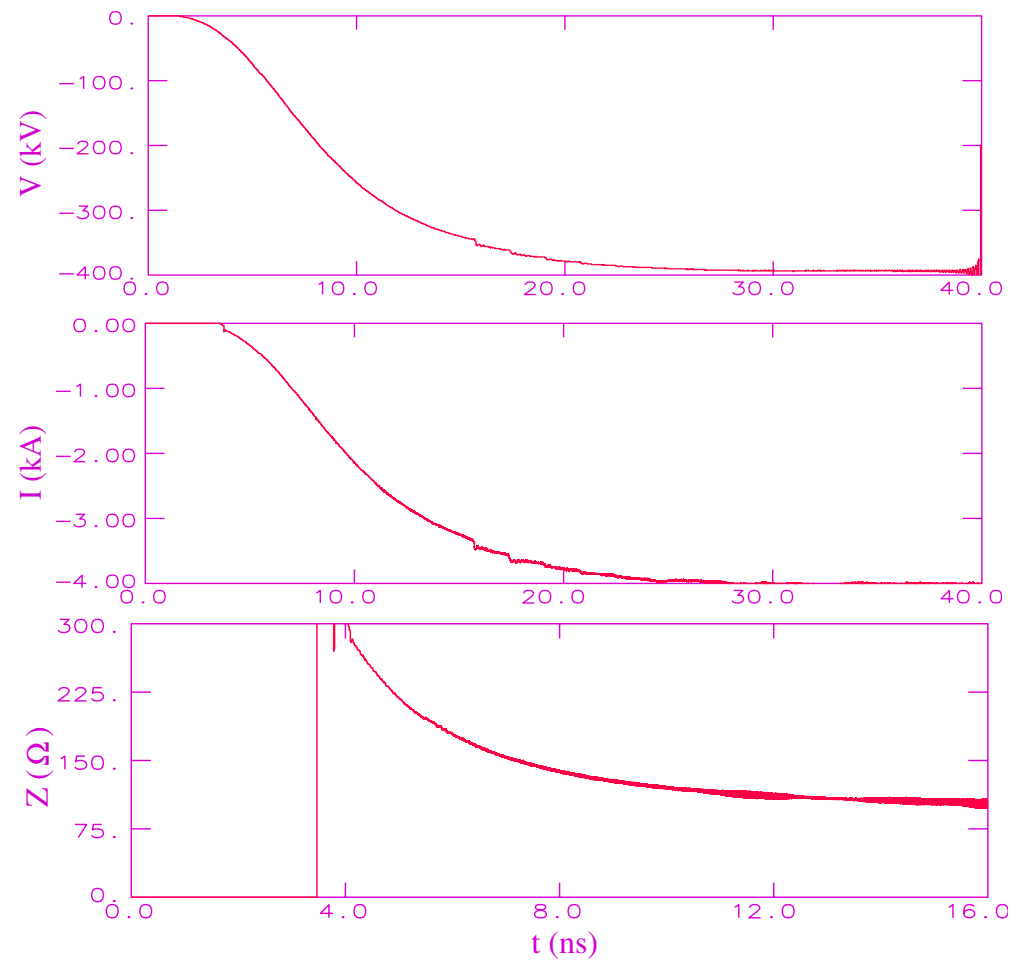

Figure 6: The diode voltage, current, and impedance for diode electron emission at $r \sim 0.61 \mathrm{~cm}$. 

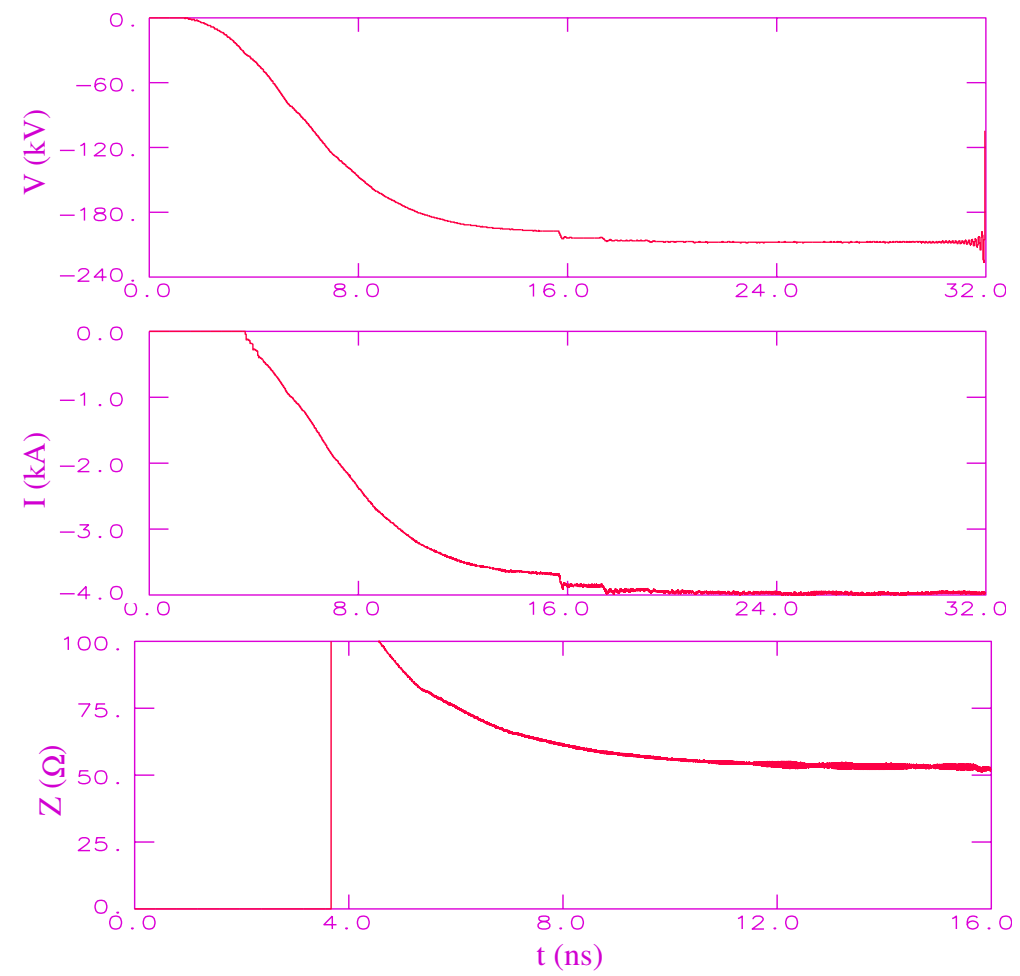

Figure 7: The diode voltage, current, and impedance for diode electron emission at $r \sim 1.67 \mathrm{~cm}$. 

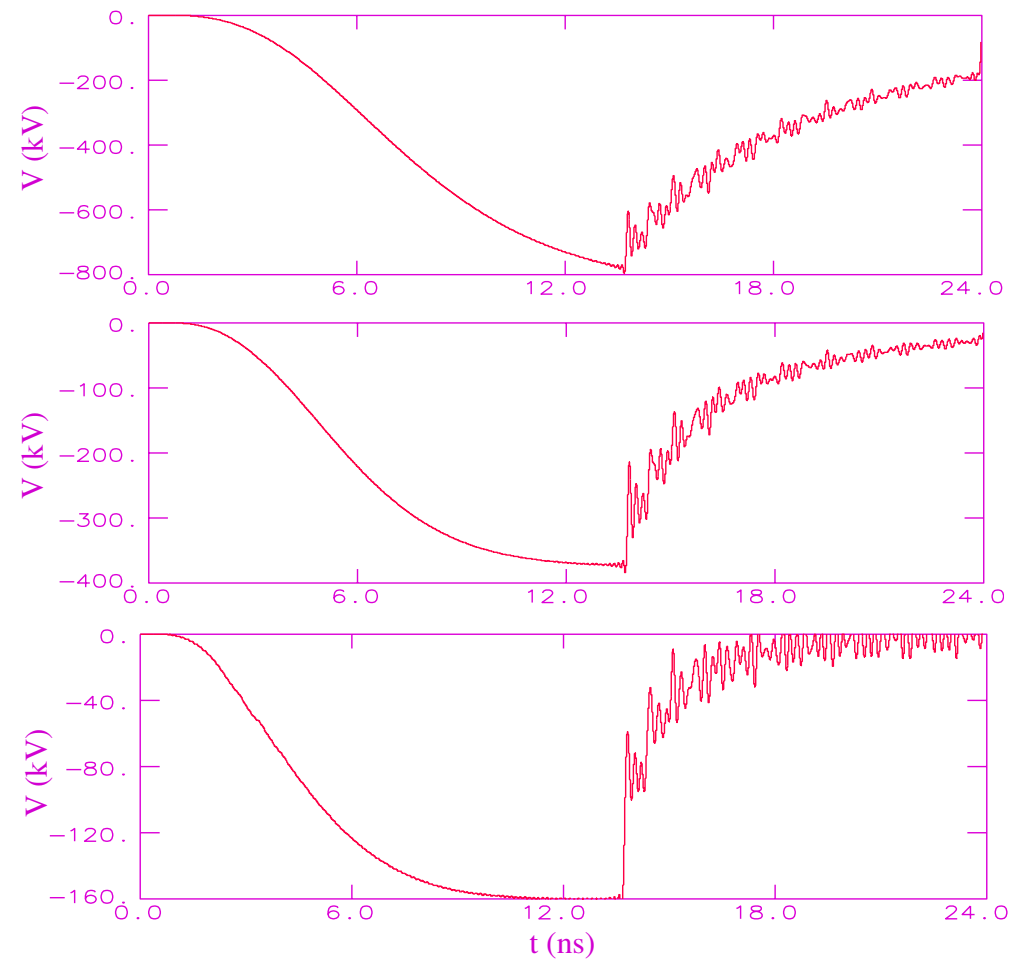

Figure 8: Simulations showing the decay of the biased potential after the beam is turned off at $t=13.2 \mathrm{~ns}$. From top to bottom: the location of diode electron emission at $r \sim 0.21$ $\mathrm{cm}, r \sim 0.61 \mathrm{~cm}$, and $r \sim 1.87 \mathrm{~cm}$. The decay time increases as the radial location of the diode gap decreases. 

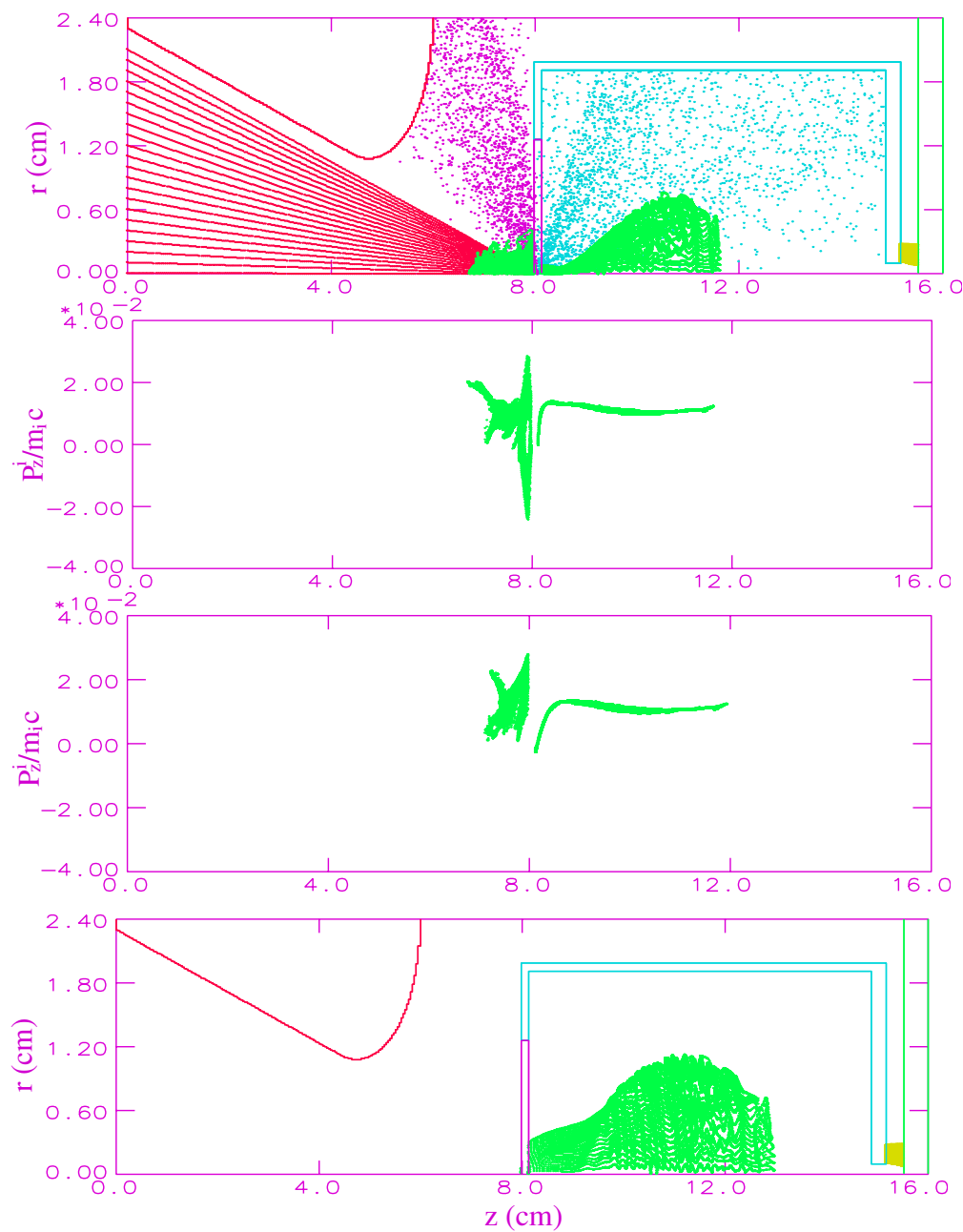

Figure 9: Results of a high biased potential run showing the re-absorption of ions by the target. The diode gap is at $r \sim 0.21 \mathrm{~cm}$ and the injection of the electron beam is stopped at $t=13.2 \mathrm{~ns}$ as the biased potential reaches nearly $800 \mathrm{kV}$. From top to bottom: ions emitted from the target at $t=13.2 \mathrm{~ns}$; axial momentum profile at $t=13.2 \mathrm{~ns}$ indicating that the ions located farther upstream have velocities pointing towards the target; axial momentum profile at $t=14.0 \mathrm{~ns}$ when the beam is absent; ions in front of the target have been re-absorbed at $t=16.5$ ns. 